\title{
Superconducting Magnet with a Minimal Steel Yoke for the Future Circular Collider Detector
}

\author{
V. I. Klyukhin ${ }^{1}$ - A. Hervé ${ }^{2}$ - A. Ball ${ }^{3} \cdot$ B. Curé $^{3} \cdot$ A. Dudarev $^{3} \cdot$ A. Gaddi $^{3}$. \\ H. Gerwig ${ }^{3}$ M. Mentink ${ }^{3}$ - H. Pais Da Silva ${ }^{3}$. G. Rolando ${ }^{3}$ - H. H. J. Ten Kate . $^{3}$ \\ C. P. Berriaud ${ }^{4}$
}

Received: 26 May 2016 / Accepted: 2 August 2016 / Published online: 17 August 2016

(C) The Author(s) 2016. This article is published with open access at Springerlink.com

\begin{abstract}
The conceptual design study of a Future Circular hadron-hadron Collider (FCC-hh) with a center-of-mass energy of the order of $100 \mathrm{TeV}$, assumed to be constructed in a new tunnel of $80-100 \mathrm{~km}$ circumference, includes the determination of the basic requirements for its detectors. A superconducting solenoid magnet of a 12-m-diameter inner bore with a central magnetic flux density of $6 \mathrm{~T}$, in combination with two superconducting dipole magnets and two conventional toroid magnets, is proposed for an FCC-hh experimental setup. The coil of $23.468 \mathrm{~m}$ length has seven 3.35-m-long modules included into one cryostat. The steel yoke with a mass of $22.6 \mathrm{kt}$ consists of two barrel layers of $0.5 \mathrm{~m}$ radial thickness and a 0.7 -m-thick nose disk and four 0.6-m-thick endcap disks each side. The outer diameter of the yoke is $17.7 \mathrm{~m}$. The full length of the magnetic system is $62.6 \mathrm{~m}$. The air gaps between the endcap disks provide for the installation of muon chambers up to an absolute pseudorapidity of about 2.7. The superconducting dipole magnets provide measurement of charged particle momenta in the absolute pseudorapidity region greater than 3 . The conventional forward muon spectrometer allows muon identification in the absolute pseudorapidity region from 2.7 to 5 . The magnet is modeled with the program TOSCA from
\end{abstract}

V. I. Klyukhin

Vyacheslav.Klyukhin@cern.ch

1 Skobeltsyn Institute of Nuclear Physics, Lomonosov Moscow State University, Moscow, 119992, Russia

2 University of Wisconsin, Madison, WI 53706, USA

3 CERN, Geneva 23, 1211, Switzerland

4 CEA Irfu, Saclay, 91191 France
Cobham CTS Limited. The total current in the superconducting solenoid coil is $123 \mathrm{MA}$ turns; the stored energy is $41.8 \mathrm{GJ}$. The axial force acting on each endcap is $450 \mathrm{MN}$. The stray field is $13.7 \mathrm{mT}$ at a radius of $50 \mathrm{~m}$ from the coil axis and $5.2 \mathrm{mT}$ at a radius of $100 \mathrm{~m}$. Many other parameters are presented and discussed.

Keywords Superconducting solenoid · Superconducting dipole magnet - Toroidal magnet

\section{Introduction}

The Future Circular hadron-hadron Collider (FCC-hh) [1] with a center-of-mass energy of the order of $100 \mathrm{TeV}$, assumed to be constructed in a new tunnel of $80-100 \mathrm{~km}$ circumference, requires in one particular experiment design the superconducting solenoid coil with a free bore of 12 $\mathrm{m}$ diameter and a central magnetic flux density of $6 \mathrm{~T}$ [1]. Future progress in tracking detector resolution will allow the momenta of prompt muons to be measured in the inner tracker, while the muon system provides identification of charged tracks as muons. In this case, the barrel part of the external muon system could be simplified using a rather thin steel yoke, with the main purpose of eliminating lowmomentum muons arising from hadron decays in flight, as well as the punch through hadrons, to ensure prompt muon identification with high purity. A magnetic flux density bending component integral of about existing Compact Muon Solenoid (CMS) value of $2.3 \mathrm{~T} \cdot \mathrm{m}$ [2] will be enough to perform this task.

The physics requirements assume the location of the major sub-detectors inside the superconducting coil. The sub-detectors are the inner tracker of $5 \mathrm{~m}$ outer diameter with a length of $16 \mathrm{~m}$, the electromagnetic calorimeter with 
an outer diameter of $7.2 \mathrm{~m}$ and a length of $18.2 \mathrm{~m}$, and the hadronic calorimeter with an outer diameter of $12 \mathrm{~m}$ and a length of at least $23 \mathrm{~m}$. The preliminary study of possible magnet design that meets these requirements is described in [3].

\section{Model Description}

The magnet system design, shown in Fig. 1, includes three major components: the superconducting coil with a total current of $123 \mathrm{MA}$ turns that creates the central magnetic flux density of $6 \mathrm{~T}$, two superconducting dipole magnets with a central magnetic flux density of $\pm 1.67 \mathrm{~T}$, and conventional toroid magnets with an averaged magnetic flux density in the steel disks of $2 \mathrm{~T}$. The superconducting solenoid coil is enclosed in a central steel magnetic flux return yoke.

\subsection{Superconducting Coil}

The superconducting solenoid coil has an inner diameter of $6.19 \mathrm{~m}$ at room temperature and a length of $23.468 \mathrm{~m}$, keeping about the same diameter-to-length ratio as that used in the CMS magnet [2, 4]. The coil consists of seven modules of $3.35 \mathrm{~m}$ long with 3 -mm-thick insulation between the modules.

To wind six layers of the coil inside the quench back cylinder of $12 \mathrm{~mm}$ thickness, made of the copper alloy, a $\mathrm{Cu}$-stabilized conductor with a cross section of $22 \times 68$

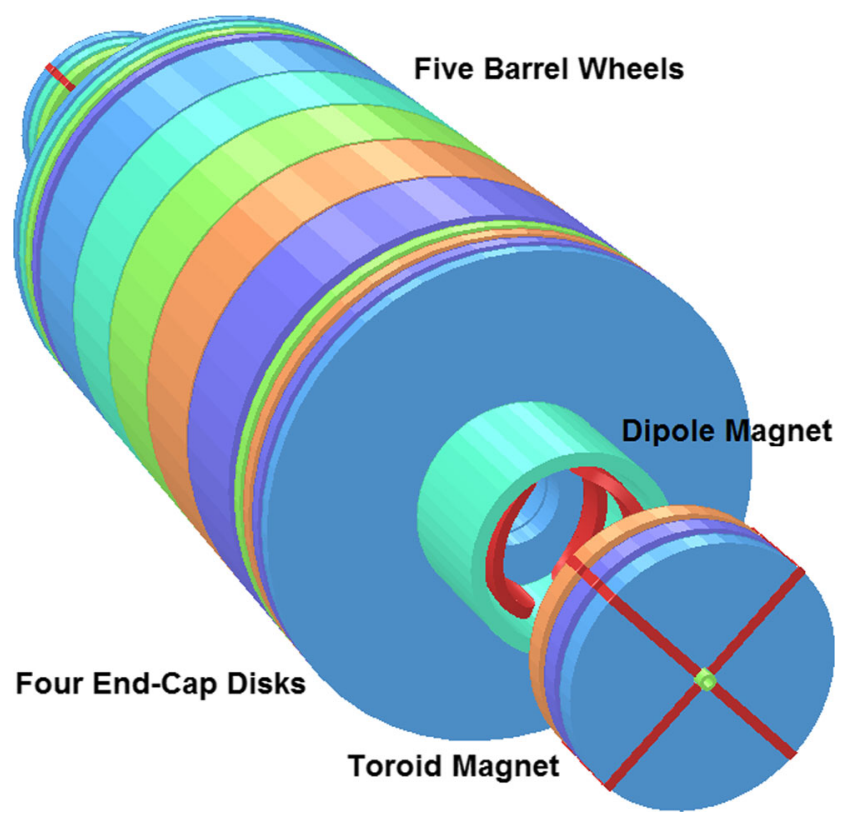

Fig. 1 Three-dimensional model of the FCC-hh detector magnetic system. Five barrel wheels, eight endcap disks, one dipole magnet, and the muon toroids are displayed. The dipole magnet coil and toroid coils are visible $\mathrm{mm}^{2}$ and NbTi superconducting insert of $2.34 \times 20.63 \mathrm{~mm}^{2}$ could be used. With a thickness of the insulation around the conductor of $0.5 \mathrm{~mm}$, the additional insulation between six coil layers of $0.4 \mathrm{~mm}$, and the insulation at the inner and outer radii of $1 \mathrm{~mm}$, the coil radial thickness with the quench back cylinder is $0.43 \mathrm{~m}$ and the conductor mass is not less than $3.39 \mathrm{kt}$.

The total number of turns is 6090, and the current corresponding to the central magnetic flux density of $6 \mathrm{~T}$ is 20.2 $\mathrm{kA}$. The stored energy in the coil $(41.8 \mathrm{GJ})$ gives a ratio of the stored energy to the coil mass of $12.3 \mathrm{~kJ} / \mathrm{kg}$ that is about the CMS value of this ratio [2, 4]. The axial pressure in the coil central plane is $84.4 \mathrm{MPa}$; the average radial pressure is $13.5 \pm 1.1 \mathrm{MPa}$, and the hoop strain is $1.89 \cdot 10^{-3}$ giving a tangential stress of $221.4 \mathrm{MPa}$.

\subsection{Central Flux Return Yoke}

The steel return yoke around the solenoid coil consists of five barrel wheels of $4.64 \mathrm{~m}$ width comprising two 0.5 $\mathrm{m}$-thick layers separated by a radial distance of $0.35 \mathrm{~m}$ as shown in Figs. 2 and 3. This gives the integral of the magnetic flux density bending component in the coil central plane of $3.86 \mathrm{~T} \cdot \mathrm{m}$ at the radial distance from 7.15 to 12 $\mathrm{m}$. To provide the sufficient value of this integral with the minimum barrel yoke thickness is the main reason of the thickness choice. The stray field is discussed in Section 3. The air gaps between the barrel wheels needed to feed out the pipes, cables, and fibers from the sub-detectors are $0.175 \mathrm{~m}$ wide.

To extend the homogenous region of the magnetic flux inside the coil, the steel nose disks with a thickness of $0.7 \mathrm{~m}$ and an outer diameter of $11 \mathrm{~m}$ are located on both sides of the coil. The distance available between the nose disks for the sub-detectors is $23.7 \mathrm{~m}$.

Four steel endcap disks of $0.6 \mathrm{~m}$ thickness connected by steel rings of $0.35 \mathrm{~m}$ thickness follow the nose disks at each coil side. The air gaps of $0.35 \mathrm{~m}$ between the barrel layers and between the endcap disks will allow the installation of the muon chambers covering the pseudorapidity [2] region of \pm 2.7 . The air gaps between the barrel wheels and the first endcap disks to provide routes for the cabling, cooling, and gas supply are $0.6 \mathrm{~m}$ wide. The axial force on each endcap is $450 \mathrm{MN}$ toward the solenoid coil center. The steel rings between the endcap disks overshadow the absolute pseudorapidity region from 2.7 to 3 , and measuring the muon momenta with the endcap muon chambers in this region is not possible.

\subsection{Superconducting Dipole Magnets}

The dipole magnets allow the charged particle momenta to be measured for absolute pseudorapidity greater than 3 . Two 


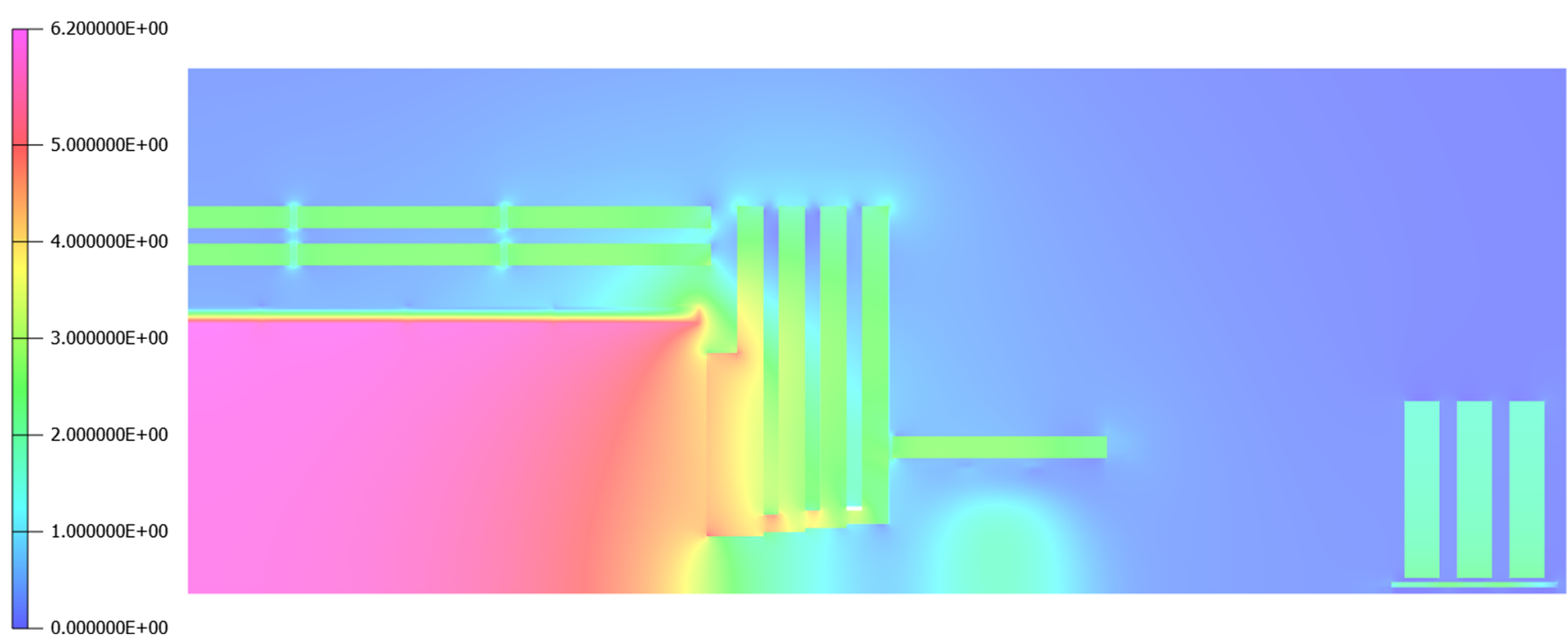

Fig. 2 Magnetic flux density distribution in the vertical plane. One quarter of the magnetic field map plotted with a cell size of $0.05 \mathrm{~m}$ has a width of $31.5 \mathrm{~m}$ and a height of $12 \mathrm{~m}$. The color scale unit is $1 \mathrm{~T}$. The minimum and maximum magnetic flux density values are 0.011 and 6.18 $\mathrm{T}$, respectively

superconducting dipole magnets one at each end of the yoke consist of a cylindrical yoke of $0.5 \mathrm{~m}$ thickness with an inner diameter of $3.1 \mathrm{~m}$ and a length of $4.9 \mathrm{~m}$ and a dipole coil split in halves with a shape of constant perimeter end with $2.25-\mathrm{m}$ inner radius. The dipole coil could be made of the same conductor as the solenoid with a cross section of $22 \times 68 \mathrm{~mm}^{2}$ and be operated with the same current of $20.2 \mathrm{kA}$ that gives a total current of 12.12 MA turns. Each coil half consists of 6 pancakes of 50 turns each. The width and the thickness of the coil at room temperature are 0.418 and $1.15 \mathrm{~m}$, respectively.

The direction of the magnetic flux density is horizontal and opposite in the two dipole magnets, and the central value of the horizontal magnetic flux density is $\pm 1.67 \mathrm{~T}$. In this case, both colliding proton beams will be deflected up. The stored energy in the dipole coil is 0.243 GJ.

The axial force on each dipole magnet is $14 \mathrm{MN}$ toward the solenoid coil center, while the horizontal force on each magnet is $-7.5 \mathrm{MN}$. The torques around the vertical axis through the dipole centers are $-76 \mathrm{MN} \cdot \mathrm{m}$ at the negative end and $76 \mathrm{MN} \cdot \mathrm{m}$ at the positive end of the central yoke.

\subsection{Conventional Toroid Magnets}

The toroid magnets provide measurements of the muons in the absolute pseudorapidity region from 2.7 to 5 . Two for-

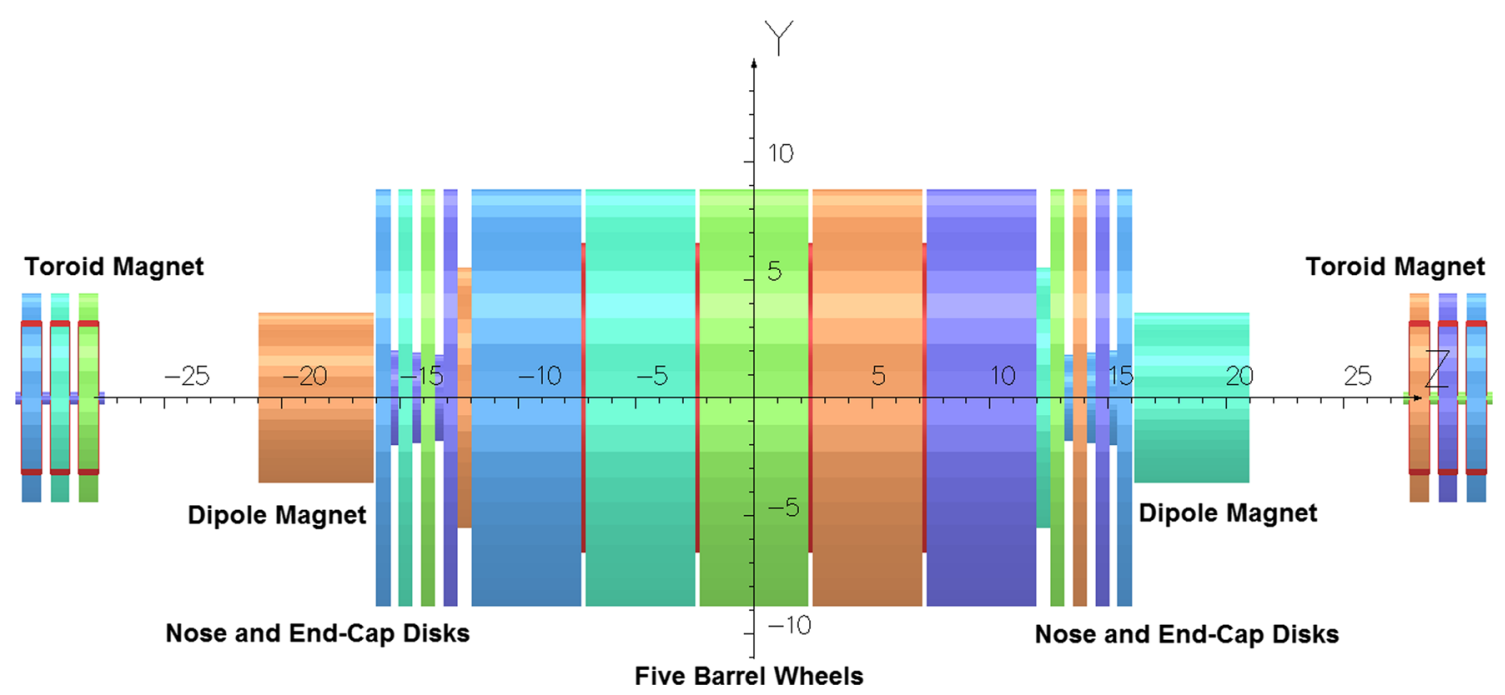

Fig. 3 The coil, the five barrel wheels of $4.64 \mathrm{~m}$ width each, the two nose disks of $11 \mathrm{~m}$ diameter each, the eight endcap disks of $17.7 \mathrm{~m}$ diameter each, the endcap rings between the disks, two dipole magnets, and the six muon toroid disks of $8.8 \mathrm{~m}$ diameter with conventional coils and protection tubes inside. The solenoid coil is visible between the barrel wheels 
ward muon spectrometers are positioned starting $27.5 \mathrm{~m}$ off the solenoid coil center at both ends of the yoke. Each spectrometer consists of three steel toroid disks of $0.8 \mathrm{~m}$ thickness with an inner diameter of $0.732 \mathrm{~m}$ and an outer diameter of $8.8 \mathrm{~m}$. There are four conventional copper coils carrying a current of 907.6 A around each toroid disk, to magnetize the steel. Each coil consists of 34 turns of $17.5 \times$ $17.5 \mathrm{~mm}^{2}$ copper conductor wound in two layers. A 10-mmdiameter hole in the conductor cross section serves for water cooling.

Still tubes with an inner diameter of $0.3 \mathrm{~m}$ and an outer diameter of $0.54 \mathrm{~m}$ keep the toroids in position, providing a gap of $0.4 \mathrm{~m}$ between the disks. The axial force on each toroid is $0.53 \mathrm{MN}$ toward the solenoid coil center. The torques around the vertical axis through the centers of three toroids are $44 \mathrm{MN} \cdot \mathrm{m}$ at the negative end and $-0.44 \mathrm{MN} \cdot \mathrm{m}$ at the positive end of the central yoke.

The total mass of steel in the magnetic system is 22.59 $\mathrm{kt}$, and the outer diameter of central yoke is $17.7 \mathrm{~m}$. The full length of the magnetic system including both forward muon spectrometers is $62.6 \mathrm{~m}$.

\section{Magnet Parameters}

In Fig. 2, the magnetic flux density distribution calculated with TOSCA [5] is displayed in the vertical plane up to a radius of $12 \mathrm{~m}$. Figure 4 shows the magnetic flux density variation along the coil axis.

Figure 5 presents the magnetic stray field variation vs. radius in the central plane of the coil. The stray magnetic flux density drops to $13.7 \mathrm{mT}$ at a radius of $50 \mathrm{~m}$ from the coil axis and to $5.2 \mathrm{mT}$ at a radius of $100 \mathrm{~m}$. For comparison, the stray field in the CMS magnet [2] at a radius of $50 \mathrm{~m}$

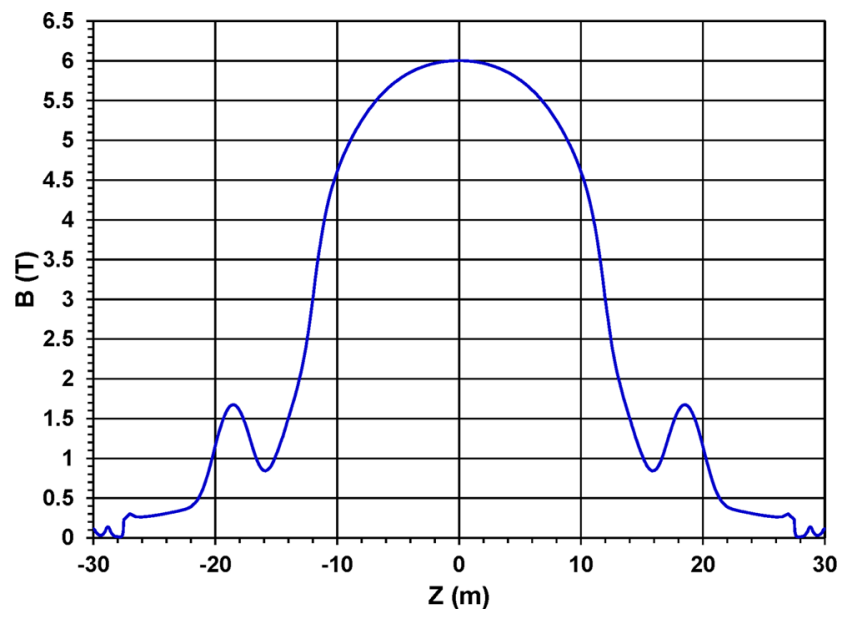

Fig. 4 Magnetic flux density variation along the coil axis

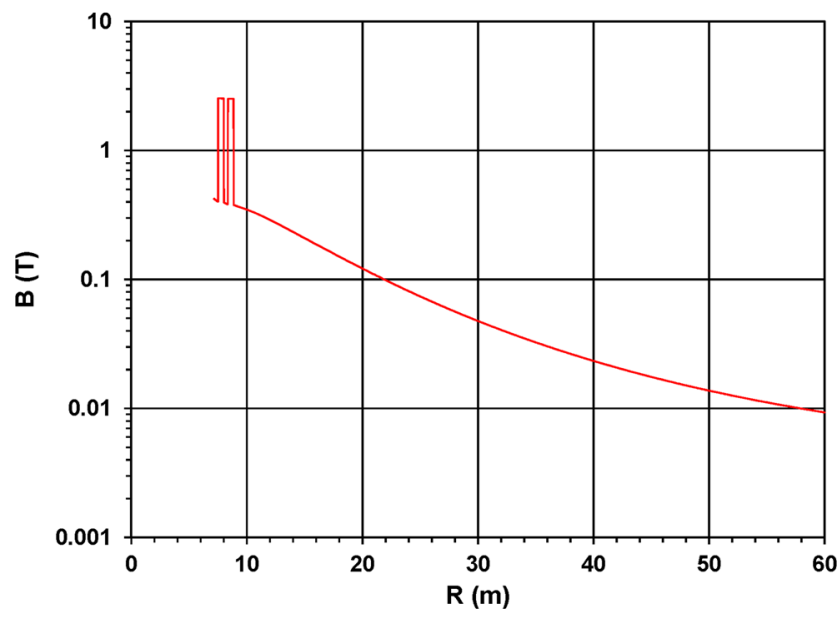

Fig. 5 Magnetic flux density out of the coil in the coil central plane vs. radius. The two peaks at about $2.5 \mathrm{~T}$ correspond to two barrel layers of the magnet flux return yoke

is $0.6 \mathrm{mT}$ comparable with the magnetic flux density of the Earth's field.

Figure 6 displays the integrals of the magnetic flux density bending component orthogonal to the charged particle trajectory vs. pseudorapidity inside the inner tracker of $5 \mathrm{~m}$ diameter and $16 \mathrm{~m}$ length and through the muon system. This plot shows that measuring the momenta of charged particles in the dipole magnets combined with muon identification in the toroid magnets complements the measurements of the momenta performed in the inner tracker within the pseudorapidity region of \pm 5 .

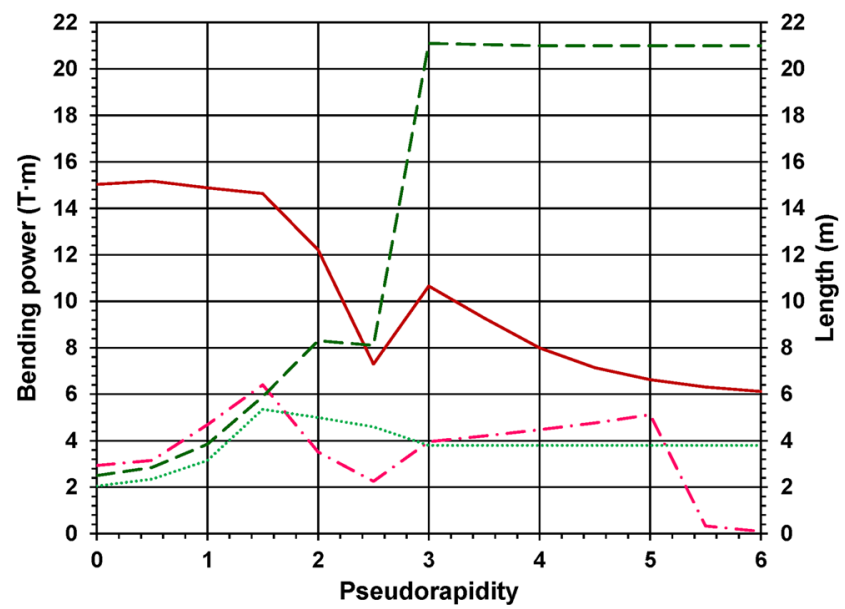

Fig. 6 Magnetic flux density bending component integrals (left scale, solid and dash-dotted lines), and the length of the charged particle trajectory (right scale, dashed and small dotted lines) in the inner tracker (smooth and dashed lines) and in the muon system (dash-dotted and small dotted lines) vs. the pseudorapidity 


\section{Conclusions}

This study investigates the idea of the superconducting solenoid magnet with a minimal steel yoke, for the Future Circular hadron-hadron Collider with a center-ofmass energy of $100 \mathrm{TeV}$. The parameters of the solenoid coil and the steel yoke seem to be technically feasible. The magnet provides the required free bore of $12 \mathrm{~m}$ diameter and a central magnetic flux density of $6 \mathrm{~T}$. The magnetic flux density distribution in the superconducting solenoid and dipole coils allow the measurement of the charged particle momenta in the pseudorapidity interval of \pm 5 . The conventional forward toroid magnets extend the region for muon identification up to the same pseudorapidity limit.
Open Access This article is distributed under the terms of the Creative Commons Attribution 4.0 International License (http:// creativecommons.org/licenses/by/4.0/), which permits unrestricted use, distribution, and reproduction in any medium, provided you give appropriate credit to the original author(s) and the source, provide a link to the Creative Commons license, and indicate if changes were made.

\section{References}

1. Future Circular Collider Study Kickoff Meeting (2014), University of Geneva - UNI MAIL, https://indico.cern.ch/event/282344/

2. CMS Collaboration: JINST 3(S08004), 1-334 (2008)

3. Klyukhin, V.I., Ball, A., Berriaud, C., Curé, B., et al.: e-print arXiv: 1511.03143 (2015)

4. Hervé, A.: Mod. Phys. Lett. A 25(20), 1647-1666 (2010)

5. TOSCA/OPERA-3d 18R2 Software, Cobham CTS Limited, Kidlington, Oxfordshire, UK 